\title{
Overabundance of Calcium in the young SNR RX J0852-4622: evidence of over-production of ${ }^{44} \mathrm{Ti}$
}

\author{
Hiroshi Tsunemi ${ }^{* \dagger}$, Emi Miyata*†, Bernd Aschenbach ${ }^{\ddagger}$ Junko Hiraga*, and Daisuke Akutsu*
}

(Received ; accepted )

\begin{abstract}
Recently, COMPTEL has detected $\gamma$-rays of $1157 \mathrm{keV}$ from ${ }^{44} \mathrm{Ti}$ in the direction of the SNR RX J0852-4622 (Iyudin et al. 1998). Since ${ }^{44} \mathrm{Ti}$ is a product of explosive nucleosynthesis and its half lifetime $\tau_{1 / 2}$ is about $60 \mathrm{yrs}$, RX J0852-4622 must be a young supernova remnant and radiation is dominated by the ejecta rather than by interstellar matter. We have detected an X-ray emission line at $4.1 \pm 0.2 \mathrm{keV}$ which is thought to come from highly ionized Ca. The emission line is so far only seen in the north-west shell region of RX J0852-4622. The X-ray spectrum can be well fitted with that of thin hot plasma of cosmic abundances except that of $\mathrm{Ca}$, which is overabundant by a factor of $8 \pm 5$. Assuming that most of $\mathrm{Ca}$ is ${ }^{44} \mathrm{Ca}$, which originates from ${ }^{44} \mathrm{Ti}$ by radioactive decay, we estimate a total Ca mass of about $1.1 \times 10^{-3} M_{\odot}$. Combining the amount of ${ }^{44} \mathrm{Ca}$ and the observed flux of the ${ }^{44} \mathrm{Ti} \gamma$-ray line, the age of RX J0852-4622 is around 1000 yrs.
\end{abstract}

Key words: ISM: individual (RX J0852-4622) — ISM: supernova remnants — X-Rays: sources - X-Rays: spectra

* Department of Earth and Space Science, Graduate School of Science, Osaka University, 1-1 Machikaneyama, Toyonaka, Osaka 560-0043 JAPAN; tsunemi@ess.sci.osaka-u.ac.jp, miyata@ess.sci.osaka-u.ac.jp, jhiraga@ess.sci.osaka-u.ac.jp, akutsu@ess.sci.osaka-u.ac.jp

$\dagger \quad$ CREST, Japan Science and Technology Corporation (JST), 2-1-6 Sengen, Tsukuba, Ibaraki 305-0047 JAPAN

$\ddagger$ Max-Planck-Institut für extraterrestrische Physik, D-85740, Garching, Germany; 


\section{Introduction}

A supernova (SN) explosion is a source of heavy elements in the Galaxy. The nucleosynthesized material inside the star is dispersed into interstellar space at relatively high temperature. In young supernova remnants (SNRs), there are many X-ray emission lines from highly ionized elements, which tend to be diluted when mixing with ambient interstellar matter.

There are two types of young SNRs from a morphological point of view: shell-like structures and those of center-filled morphology. There are also two types of young SNRs from the spectroscopic point of view: thin thermal emission and power-law type spectra. The Crab nebula, a young SNR, shows a center-filled structure with a power-law type spectrum. There is a neutron star, the Crab pulsar, in its center, which is the energy source of the SNR. There are more SNRs showing shell-like structures with spectra due to optically thin thermal emission, e.g. Cassiopeia-A, Tycho and Kepler's SNRs. The shell structure is the result of the interaction of the shock waves and corresponding shock-heating of the ambient plasma. This produces thin thermal emission including many emission lines. We note that the SNe of Tycho and Kepler have been witnessed and recorded in historical documents whereas there is no record of Cassiopeia-A which is estimated to be born late in the 17th century.

The remnant of the SN which occurred in AD1006, SN1006, is another young SNR. It shows a pronounced shell-like structure. Koyama et al. (1996) revealed that the spectra from the north-east and south-west regions of the shell are of power-law type while that from the interior is thin thermal emission. They claimed that the shell region in SN1006 is a site of cosmic ray shock acceleration with energies of up to $200 \mathrm{TeV}$. Accordingly, the shell-like structure does not always stand for a thermally radiating SNR.

Cassiopeia-A, one of the young SNRs, is the first source from which $\gamma$-ray emission lines from ${ }^{44} \mathrm{Ti}$ have been reported (Iyudin et al. 1994). ${ }^{44} \mathrm{Ti}$ is expected to be produced in the explosive nucleosynthesis inside the SN which also produces ${ }^{56} \mathrm{Ni} .{ }^{44} \mathrm{Ti}$ decays into ${ }^{44} \mathrm{Sc}$ emitting two hard $\mathrm{X}$-ray lines of $68 \mathrm{keV}$ and $78 \mathrm{keV}$ with a half lifetime, $\tau_{1 / 2}$, of $60 \mathrm{yrs}$ (Ahmad et al. 1998). ${ }^{44} \mathrm{Sc}$ decays further to ${ }^{44} \mathrm{Ca}$ emitting a $\gamma$-ray line at $1157 \mathrm{keV}$ with $\tau_{1 / 2}$ of 3.9 hours which has been detected with COMPTEL. In this way, ${ }^{44} \mathrm{Ti}$ is converted into ${ }^{44} \mathrm{Ca}$. Taking into account the short lifetime of ${ }^{44} \mathrm{Ti}$, its detection is direct evidence 
of the source being a young SNR. The discovery of this second celestial source of ${ }^{44} \mathrm{Ti}$ has been reported by Iyudin et al. (1998): it is located in the direction of the south-east part of the Vela SNR where Aschenbach (1998) discovered a new independent SNR, RX J0852-4622, which has a radius of $\simeq 1^{\circ}$. The distance to this source is not clear while it is on the way to the Vela SNR that is $250 \mathrm{pc}$ away (Cha et al. 1999). Based on the X-ray and ${ }^{44} \mathrm{Ti}$ data, RX J0852-4622 is expected to be born several hundred yrs ago in a sky region which in principle could have been observed from China, and therefore some record in a historical document is expected if the SN was of standard brightness. However, if it were sub-luminous the event might have been missed by the contemporaries (Aschenbach et al. 1999). So far, no record has been found, which reminds us of some similarity with Cassiopeia-A. We note that quite recently a footprint of a historical SN has been found in terms of nitrate abundance in Antarctic ice cores (Burgess \& Zuber 1999). The associated SN would have occurred around $1320 \pm 30$ yrs.

We have observed RX J0852-4622 with ASCA on December 21-24, 1998 as a TOO target. This observation was performed with the two CCD cameras (SIS) (Yamashita et al. 1997) and the two imaging gas scintillation proportional counters (GIS) (Makishima et al. 1996) which are placed at the focal plane of four thin-foil X-ray mirrors (Serlemitsos et al. 1995) on board the ASCA satellite (Tanaka et al. 1994). In this paper, we concentrate on the emission from the north-west bright shell region. The entire remnant will be reported elsewhere.

\section{Observation and Data Screening}

The observation was performed in 7 pointings so that we could cover the entire remnant with the GIS. Figure 1 shows the GIS field of view (FOV) superimposed on the ROSAT all-sky survey image (Aschenbach et al. 1999). We should note that we did not cover the south bright shell region. The observational $\log$ is shown in table 1 . We excluded all the GIS data taken at elevation angle below $5^{\circ}$ from the night earth rim and $25^{\circ}$ from the day earth rim, a geomagnetic cutoff rigidity lower than $6 \mathrm{GeV}$ $\mathrm{c}^{-1}$, and the region of the South Atlantic Anomaly (SAA). We also applied a "flare-cut" to maximize the signal-to-noise ratio described in Ishisaki et al. within the frame work of ASCA_ANL (Ishisaki Y. et al, 1997, ASCA News letter, No.5 ). In this way, the effective exposure time is $\simeq 76 \mathrm{ksec}$ after the 
data screening. We constructed three kinds of images for the GIS data set: a total count image, a non-X-ray background image, and a cosmic X-ray background image. As shown in table 1, the GIS bit assignments are slightly different from pointing to pointing, the binsize of coordinates is different. We redistributed events in each binning area with a Poisson random number to make images having the same dimensions. The non-X-ray background image was generated with the H02-sorting method in DISPLAY45 (the detailed description of this method is in Ishisaki (1996)). The image of the cosmic X-ray background was extracted from the Large Sky Survey (LSS) data observed during the ASCA PV phase (Ueda et al. 1999). We also extracted the non-X-ray background image from the LSS data to produce the mean cosmic X-ray background image solely. Thus, we extracted background-subtracted GIS images in the energy band of $0.7-10 \mathrm{keV}$ as shown in figure 2. We can find a well-defined shell structure and a compact point-like source at the geometrical center of the X-ray shell.

In the case of the SIS data analysis, we excluded all the data taken at elevation angle below $5^{\circ}$ from the night earth rim and $30^{\circ}$ from the day earth rim, a geomagnetic cutoff rigidity lower than 6 $\mathrm{GeV} \mathrm{c}^{-1}$, the region from $128 \mathrm{~s}$ before entering the SAA to the end of the SAA, and the period of $120 \mathrm{~s}$ at the day-night change of the satellite. After the screening, the total exposure time is $\simeq 59 \mathrm{ks}$. We, next, manually removed the time region where we could see a sudden change in the light curves of the corner pixels of X-ray events. Then, we removed the hot and flickering pixels and corrected CTI, DFE, Echo effects, and RDD (T. Dotani et al. 1995, ASCA News Letter 3, 25; T. Dotani et al. 1997, ASCA Letter News 5,14$)$ in our data sets. We employed the calibration file of sisph2pi_110397.fits for CTI corrections. For RDD corrections, we need to look for the frame data obtained near to the epoch

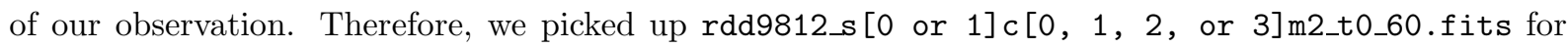
RDD corrections retrieved from the Goddard Space Flight Center anonymous ftp site.

\section{Spectrum Analysis}

The GIS observation shows a shell-like structure similar to that obtained with ROSAT. Its X-ray spectrum is almost uniform in spectral shape over the remnant, consisting of two components: a power-law component with a photon index, $\Gamma$, of $\sim 2.5$ and thin thermal emission of electron temperature, $k T_{\mathrm{e}}$, of 
$0.07-0.21 \mathrm{keV}$. The X-ray spectrum from the entire region is shown in figure 3. RX J0852-4622 overlaps with the Vela SNR that emits thin thermal emission with $k T_{\mathrm{e}}$ of about $0.17 \mathrm{keV}$. This value is very close to that of the low temperature component seen in RX J0852-4622. Furthermore, we find the intensity of this component to be very similar to that appeared in the Vela SNR component around RX J0852-4622. We assume that the low temperature component seen in RX J0852-4622 is background emission from the Vela SNR. Therefore we can expect that the emission from RX J0852-4622 is predominantly of the power-law type originating from a shell-like region.

The SIS can cover only the central region of each GIS FOV. Among them, the spectrum of the bright north-west shell clearly shows features which differ from those observed elsewhere as shown in figure 4 . We see a clear emission line structure around $4 \mathrm{keV}$. We see this structure only in the eastern part of the north-west bright shell region. Other regions covered with the SIS show featureless spectra similar to that obtained with the GIS for the entire remnant. For comparison, figure 5 shows the SIS spectrum extracted from the rectangular region shown with the broken line in figure 1 . There is no clear emission line structure around $4 \mathrm{keV}$. This spectrum was well represented by a thin thermal emission with $k T_{\mathrm{e}}$ of $0.10_{-0.02}^{+0.01} \mathrm{keV}$ adding a power-law spectrum with $\Gamma$ of $2.8_{-0.2}^{+0.1}$. Quoted errors in this paper are at $90 \%$ confidence level.

We fitted the SIS spectrum of the north-west shell with a two-component model. First of all, we employed a low $k T_{\mathrm{e}}$ thin thermal emission model with cosmic abundance (mekal) in order to represent the emission from the Vela SNR. We then added another component for the emission from RX J0852-4622 described below. The neutral hydrogen column density, $N_{\mathrm{H}}$, of two components were set to be equal as one free parameter.

We applied a thermal bremsstrahlung model for the emission from RX J0852-4622 proper, but we could not obtain an acceptable fit as shown in model-1 of table 2. The big discrepancy between data and model is found around $4 \mathrm{keV}$. We thus added a Gaussian component to reproduce the structure around $4 \mathrm{keV}$ (model-2). In this fitting, we fixed sigma of Gaussian to be 0 . Then, $\chi^{2}$ is improved by $\sim 10$ compared with the model-1 shown in table 2. If we apply the F-test, the significance level of an additional $4 \mathrm{keV}$ line is $\sim 99 \%$. The line center energy is $4.1 \pm 0.2 \mathrm{keV}$ and flux is $(5 \pm 2) \times 10^{-5}$ counts 
$\mathrm{s}^{-1} \mathrm{~cm}^{-2}$ over the SIS FOV.

We then employed the thin thermal model with variable cosmic abundance (vmekal) (model-3). Free parameters are $k T_{\mathrm{e}}$, the normalization of two thermal emission components, the abundances of $\mathrm{Si}$, $\mathrm{S}$, and $\mathrm{Ca}$ of the high $k T_{\mathrm{e}}$ component, and $N_{\mathrm{H}}$. Abundances of the other elements are fixed to cosmic values. This model is acceptable at $90 \%$ confidence level. The Ca abundance is $8 \pm 5$ times larger than the cosmic value. The absolute value is less meaningful since it strongly depends on the applied model and other model parameters. However, the relative abundances among the elements are relatively robust. As shown in table 2, the Ca abundance is 11 times and 40 times larger than those of Si and S, respectively.

If we employ a thin thermal model with nonequilibrium collisional ionization (NEI) (Masai 1984; Masai 1994), $k T_{\mathrm{e}}$ increases while the metal abundances do not change within the statistical uncertainties as shown in table 2 (model-4).

\section{Discussion}

There are some candidates for the $4.1 \pm 0.2 \mathrm{keV}$ emission line from an astrophysical point of view. If it is a characteristic X-ray line, it must be either from neutral Sc-K or a helium-like Ca-K emission line. From the cosmic abundance point of view, it is very implausible that it comes from Sc. If it comes from shock-heated matter, it is plausible that it comes from Ca at high temperatures. If this is not the case, it can be either a red-shifted Fe-K line from an AGN or a cyclotron emission line from a strongly magnetized neutron star. Since the line emission region is quite extended judging from the ASCA image, none of the hypotheses of a point-like source is very likely.

The overabundance of $\mathrm{Ca}$ can be a result of an overabundance of ${ }^{44} \mathrm{Ti}$ which is produced only in the SN explosive nucleosynthesis process. It is mainly produced deep in the interior of the star, both

in a type Ia and type II SNe. In the following we assume that the ejecta containing ${ }^{44} \mathrm{Ti}$ are uniformly expanding into ambient space. In the early phase of the SNR evolution, the major part of the emission does not come from the shock heating process but comes from accelerated and decelerating particles, which produce a power-law type X-ray spectrum. In the north-west bright shell region, the ejecta might have recently collided with an interstellar cloud forming shock-heated thermal plasma, which is the source 
of the Ca emission line.

The expanding ejecta happen to hit the interstellar cloud at the north-west shell. The ejecta expanding along other directions should also contain similar amounts of Ca but they may not yet been shock-heated, so that just the power-law type spectrum prevails with no emission lines. We note that there is another bright shell region in the south, which we did not observe in our ASCA observations.

Based on the emission line detected, we can estimate the total amount of Ca. The north west bright shell region is about $11^{\prime}$ square where we detected the emission line. We assume that the depth of this region is as equal to the width. Assuming that the filling factor of this region is unity, we find that the plasma density in this region is $(0.9 \pm 0.1)(D / 200 \mathrm{pc})^{-0.5} \mathrm{H} \mathrm{cm}^{-3}$ where $D$ is the distance to the source, resulting a total mass to be $5.4 \times 10^{-3} M_{\odot}$. Then, we can evaluate the total mass in all directions assuming spherical symmetry. In this way, we obtained the total amount of $\mathrm{Ca}, M_{\mathrm{Ca}}$, contained in $\mathrm{RX}$ J0852-4622 to be $1.1 \times 10^{-3} M_{\odot}(D / 200 \mathrm{pc})^{2.5}(A / 8)$, where $A$ is the abundance of Ca. We assume that all the ${ }^{44} \mathrm{Ca}$ is in the shell region but only that fraction expanding towards the north-west shell is actually shock-heated and visible in the Ca line.

The major isotope of $\mathrm{Ca}$ on Earth is ${ }^{40} \mathrm{Ca}$ while the fraction of ${ }^{44} \mathrm{Ca}$ is about $2 \times 10^{-2}$ (Anders \& Grevesse 1989). Assuming that the other isotopes of Ca are produced with the abundances similar to the terrestrial values, ${ }^{44} \mathrm{Ca}$, the product of ${ }^{44} \mathrm{Ti}$, should be heavily over-abundant, and we expect that almost all the Ca detected must be ${ }^{44} \mathrm{Ca}$.

Based on the theoretical models of Nomoto et al. (1984) and Thielemann et al. (1996), type II SN can produce Ca of $5 \times 10^{-3} M_{\odot}$, of which value slightly depends on the progenitor star mass, whereas type I SN can produce $1.2 \times 10^{-2} M_{\odot}$. Since we do not know whether we have detected all Ca in RX J0852-4622, it is inappropriate to compare our results with those of the model calculations. In both cases, the mass ratio of $\mathrm{Ca}$ to $\mathrm{Si}$ is less or similar to that of the cosmic value. These models cannot explain the overabundance of Ca. Whereas these models assume the point symmetric explosion, asymmetric explosions can produce large metal abundance anomalies (Nagataki et al. 1997), which may explain the overabundance of Ca. If we assume that most of Ca comes from ${ }^{44} \mathrm{Ti}$, we can estimate the age, $t$, of RX J0852-4622 using the observed flux, $F$, of the $1157 \mathrm{keV} \gamma$-rays. We obtain $\mathrm{t} / \tau_{1 / 2}=16-\log _{2}$ 
$\left(\left(F / 3.8 \times 10^{-5}\right.\right.$ photon $\left.\left.\mathrm{cm}^{-2} \mathrm{~s}^{-1}\right)(D / 200 \mathrm{pc})^{1 / 2}(A / 8)\right)$. This value results in an upper limit of the age of $970 \mathrm{yrs}$ and the lowest limit for the expansion velocity of $2900 \mathrm{~km} \mathrm{~s}^{-1}$. Since there occurs shock-heating associated with deceleration of the expansion, the initial expansion velocity must have been higher than this value. If we assume that $\mathrm{Ca}$ in $\mathrm{RX}$ J0852-4622 has an isotope population similar to that of the terrestrial value, the mass of ${ }^{44} \mathrm{Ti}$ is reduced by a factor of 50 . Then we obtain a lower limit of the age of $630 \mathrm{yrs}$ and an upper limit of the expansion velocity of $4500 \mathrm{~km} \mathrm{~s}^{-1}$.

Model calculations of SNe show that the heavy elements are produced relatively deep inside the progenitor star. In type II SN, ${ }^{44} \mathrm{Ti}$ is produced very close to the mass-cut point, which is the critical boundary separating the ejecta and the mass forming a central compact remnant. If the SN explosion occurs in a homologous fashion, ${ }^{44} \mathrm{Ti}$ and $\mathrm{Ca}$ are concentrated in a narrow layer. We can expect that the entire $\mathrm{Ca}$ is shock-heated simultaneously. Based on the SN model calculations the expanding velocity of the Ca dominated layer is a few thousand $\mathrm{km} \mathrm{s}^{-1}$ (Shigeyama et al. 1988). Therefore, the age of RX J0852-4622 is likely to be closer to 970 yrs rather than 630 yrs.

\section{Conclusion}

We observed the SNR, RX J0852-4622, that is the ${ }^{44} \mathrm{Ti} \gamma$-ray source. It is towards the direction to the Vela SNR that is $250 \mathrm{pc}$ away from us. Based on the spectral analysis, the apparent spectrum from the entire remnant consists of two components; one is a power-law component and the other is thermal emission coming from the Vela SNR.

In the north-west bright shell, we have detected an emission line feature. This feature is only seen by the SIS over a small fraction of the remnant. The line center is $4.1 \pm 0.2 \mathrm{keV}$ which is interpreted as an emission line from highly ionized $\mathrm{Ca}$. We find that the $\mathrm{Ca}$ abundance in this region is about $8 \pm 5$ times that of the cosmic value while the other elements are subcosmic.

If the Ca abundance anomaly mainly comes from the overabundance of ${ }^{44} \mathrm{Ca}$ that is produced from

${ }^{44} \mathrm{Ti}$, we can evaluate the age of this source using the flux of the ${ }^{44} \mathrm{Ti} \gamma$-rays. Since we cannot distinguish among the Ca isotopes, the age estimate is between $630 \mathrm{yrs}$ and $970 \mathrm{yrs}$. If the Ca isotopes except ${ }^{44} \mathrm{Ca}$ are produced at cosmic abundance levels, the age is $970 \mathrm{yrs}$. 
We thank ASCA_ANL and DISPLAY 45 developing team members, especially Dr. Ishisaki. We are grateful to all the members of ASCA team for their contributions to the fabrication of the apparatus, the operation of ASCA, and the data acquisition. This research is partially supported by the Sumitomo Foundation. 


\section{References}

Ahmad I, Bonino G., Castagnoli G.C., Fischer S.M., Kutschera W., Paul M. 1998, Phys. Rev. Lett. 80, 2550

Anders E., Grevesse N. 1989, Geochim. Cosmochim. Acta, 53, 197

Aschenbach B. 1998, Nature, 396, 141

Aschenbach B., Iyudin A.F., Schönfelder V., 1999, A\&A, 350, 997

Burgess C. P. Zuber K. 1999, astro-ph/9909010

Cha R.N., Sembach K.R. Danks A.N. 1999, ApJ, 515, L25

Ishisaki Y. 1996, Ph.D. thesis of Univ. of Tokyo, ISAS RN 613

Iyudin A.F., Schönfelder V., Bennett K., Bloemen H., Diehl R., Hermsen W., Lichti G.G., van der Meulen R.D. et al. 1998, Nature, 396, 142

Iyudin A.F., Diehl R., Bloemen H., Hermsen W., Lichti G.G., Morris D., Ryan J., Schönfelder V. et al. 1994, A\&A, 284, L1

Koyama K., Petre R., Gotthelf E.V., Hwang U. Matsuura M., Ozaki M., Holt S.S. 1995, Nature, 378, 255

Makishima K., Tashiro M., Ebisawa K., Ezawa H., Fukazawa Y., Gunji S., Hirayama M., Idesawa E. et al. 1996, PASJ 48, 171

Mewe R., Gronenschild E.H.B.M., van den Oord G.H.J., 1985, A\&AS, 62, 197

Masai K., 1984, Ap\&SS 98, 267

Masai K. 1994, Ap.J., 437, 770

Nagataki S., Hashimoto M., Sato K. Yamada S. 1997, ApJ, 486, 1026

Nomoto K., Thielemann F.-K., Yokoi K., 1984, ApJ, 286, 644

Serlemitsos P.J., Jalota L., Soong Y., Kunieda H., Tawara Y., Tsusaka Y., Suzuki H., Sakima Y. et al. 1995, PASJ 47, 105

Shigeyama T., Nomoto K., Hashimoto M. 1988, A\&Ap, 196, 141

Tanaka Y., Inoue H., Holt S.S. 1994, PASJ 46, L37

The L.-S., Leising M.D., Clayton D.D., Johnson W.N., Kinzer R.L., Kurfess J.D., Strickman M.S., Jung G.V. et al. 1995, ApJ, 444, 244 
Thielemann F.-K., Nomoto K., Hashimoto M., 1996, ApJ, 460, 408

Ueda Y., Takahashi T., Inoue H., Tsuru T., Sakano M., Ishisaki Y., Ogasaka Y., Makishima K. et al. 1999, ApJ, 518, 656

Yamashita A., Dotani T., Bautz M., Crew G., Ezuka H., Gendreau K., Kotani T., Mitsuda K. et al. 1997, IEEE Trans. Nuc. Sci. 44, 847

Yamashita A., Dotani T., Ezuka H., Kawasaki M., Takahashi K. 1999, Nuclear Inst. Method A, 436, 68 
Fig. 1. The circular FOVs of the GIS are superimposed on the X-ray intensity map obatained with ROSAT. There are two bright shell regions in the north and south.

Fig. 2. X-ray surface brightness map obtained with the ASCA GIS. The GIS image is smoothed with a Gaussian of $\sigma=30^{\prime \prime}$ after rebinning of $2 \times 2\left(30^{\prime \prime}\right.$ square) pixels.

Fig. 3. The X-ray spectrum from the entire remnant obtained with the GIS

Fig. 4. The X-ray spectrum from the white square shown in figure 1. Solid lines represent the best fit curve of model-3 (see text). Ca is overabundant by $8 \pm 5$.

Fig. 5. The X-ray spectrum from the rectangular region shown with the broken line in figure 1. Solid lines represent the best fit curve of the model of thin thermal emission adding a power-law spectrum. 
Table 1. Observational Log

\begin{tabular}{|c|c|c|c|c|c|}
\hline Pointing ID & RA (J2000) & Decl.(J2000) & SIS chips & GIS bit assignment* & Exposure time $\dagger$ \\
\hline $\mathrm{N} 1 \ldots \ldots \ldots$ & $8^{\mathrm{h}} 49^{\mathrm{m}} 18^{\mathrm{s}} .1$ & $-45^{\mathrm{d}} 41^{\prime} 0^{\prime \prime}$ & S0C12, S1C30 & $8-6-6-5-0-6$ & 9.3 \\
\hline $\mathrm{N} 2 \ldots \ldots \ldots$ & $8^{\mathrm{h}} 53^{\mathrm{m}} 42^{\mathrm{s}} .4$ & $-45^{\mathrm{d}} 32^{\prime} 29^{\prime \prime}$ & S0C01, S1C01 & $8-6-6-0-0-10$ & 10.4 \\
\hline $\mathrm{N} 3 \ldots \ldots \ldots$ & $8^{\mathrm{h}} 55^{\mathrm{m}} 31.2$ & $-45^{\mathrm{d}} 57^{\prime} 12^{\prime \prime}$ & S0C12, S1C30 & $8-6-6-5-0-6$ & 13.7 \\
\hline N4 . & $8^{\mathrm{h}} 51^{\mathrm{m}} 43.2$ & $-46^{\mathrm{d}} 7^{\prime} 31^{\prime \prime}$ & S0C12, S1C30 & $8-6-6-5-0-6$ & 11.3 \\
\hline N5 ........ & $8^{\mathrm{h}} 47^{\mathrm{m}} 47^{\mathrm{s}} .1$ & $-46^{\mathrm{d}} 18^{\prime} 5^{\prime \prime}$ & $\mathrm{S} 0 \mathrm{C} 12, \mathrm{~S} 1 \mathrm{C} 30$ & $8-6-6-5-0-6$ & 9.8 \\
\hline $\mathrm{N} 6 \ldots \ldots \ldots$ & $8^{\mathrm{h}} 51^{\mathrm{m}} 6.6$ & $-46^{\mathrm{d}} 46^{\prime} 58^{\prime \prime}$ & S0C12, S1C30 & $8-6-6-5-0-6$ & 11.9 \\
\hline N7 ........ & $8^{\mathrm{h}} 54^{\mathrm{m}} 50.9$ & $-46^{\mathrm{d}} 32^{\prime} 17^{\prime \prime}$ & S0C12, S1C30 & $8-6-6-5-0-6$ & 9.8 \\
\hline
\end{tabular}

* Each field stands for bit number of a pulse-height, $\mathrm{x}$ coordinate, y coordinate, rise-time, spread discrimination, and timing.

$\dagger$ Exposure time of the GIS is shown. 
Table 2. Fitting results of the SIS spectrum at the north-west region.

\begin{tabular}{lccccccc}
\hline \hline & & & & & & & \\
model & low $k T_{\mathrm{e}}$ & high $k T_{\mathrm{e}}$ & $\mathrm{Si}$ & $\mathrm{S}$ & $\mathrm{Ca}$ & $N_{\mathrm{H}}$ & $\chi^{2}(\mathrm{dof})$ \\
& $(\mathrm{keV})$ & $(\mathrm{keV})$ & & & & $\left(10^{22} \mathrm{~cm}^{-2}\right)$ & \\
\hline & & & & & & & \\
model-1 & $\left(3.28_{-0.66}^{+0.04}\right) \times 10^{-2}$ & $2.0_{-0.3}^{+0.4}$ & - & - & - & $0.12_{-0.05}^{+0.09}$ & $99.3(81)$ \\
model-2 & $\left(3.29_{-0.63}^{+0.04}\right) \times 10^{-2}$ & $1.8 \pm 0.3$ & - & - & - & $0.14_{-0.06}^{+0.09}$ & $89.2(79)$ \\
model-3 & $\left(4.25_{-0.05}^{+0.70}\right) \times 10^{-2}$ & $1.32 \pm 0.09$ & $0.7 \pm 0.4$ & $\leq 0.2$ & $8 \pm 5$ & $1.1 \pm 0.1$ & $87.2(78)$ \\
model-4 & $(5 \pm 1) \times 10^{-2}$ & $3.1_{-0.8}^{+1.6}$ & $0.9_{-0.2}^{+0.3}$ & $\leq 0.1$ & $7_{-5}^{+7}$ & $0.83 \pm 0.1$ & $88.9(77)$ \\
\hline
\end{tabular}

Quoted errors are at $90 \%$ confidence level.

* Obtained ionization parameter is $10.8 \pm 0.1$. 
This figure "fig1.jpg" is available in "jpg" format from: http://arxiv.org/ps/astro-ph/0005452v1 
This figure "fig2.jpg" is available in "jpg" format from: http://arxiv.org/ps/astro-ph/0005452v1 


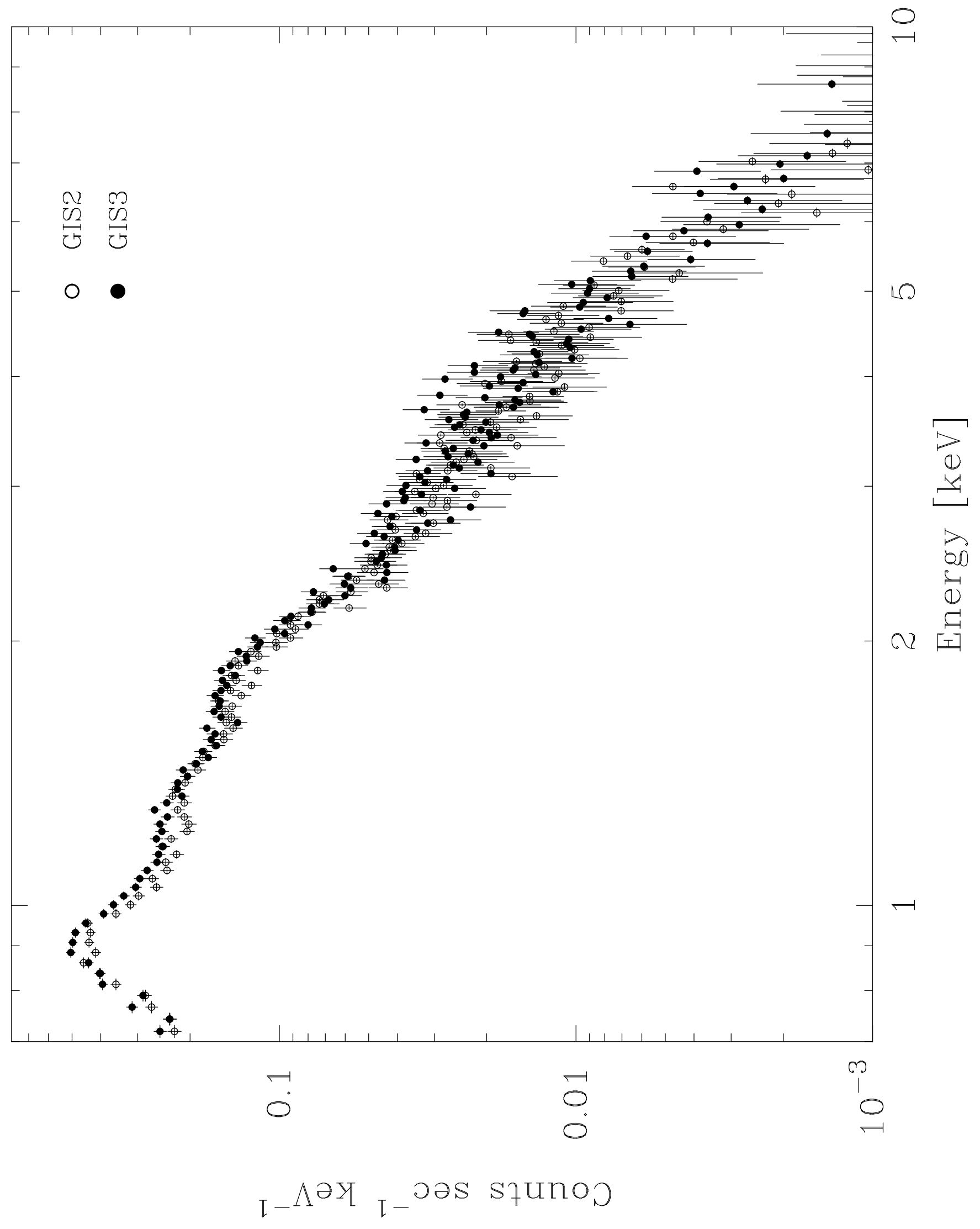




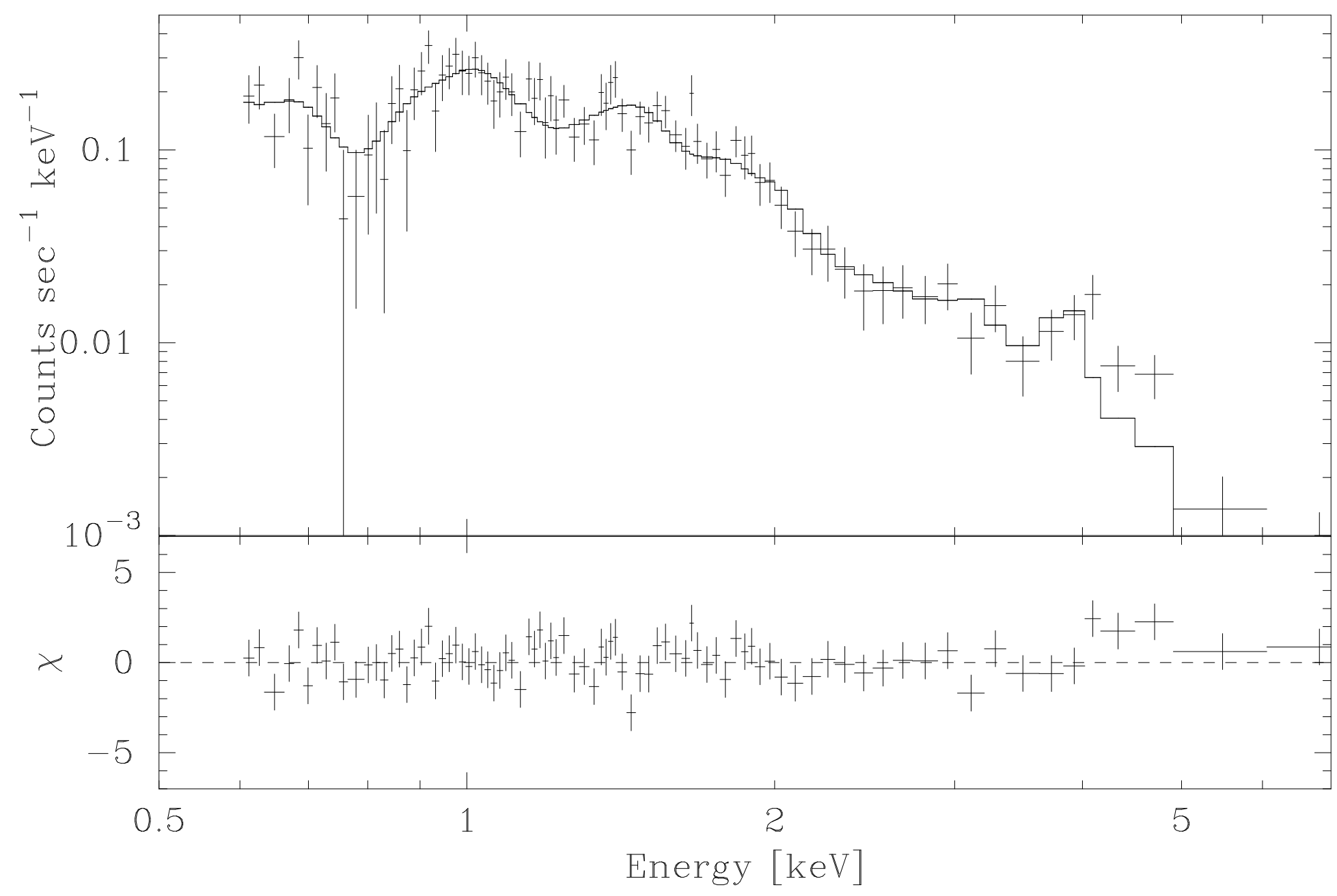




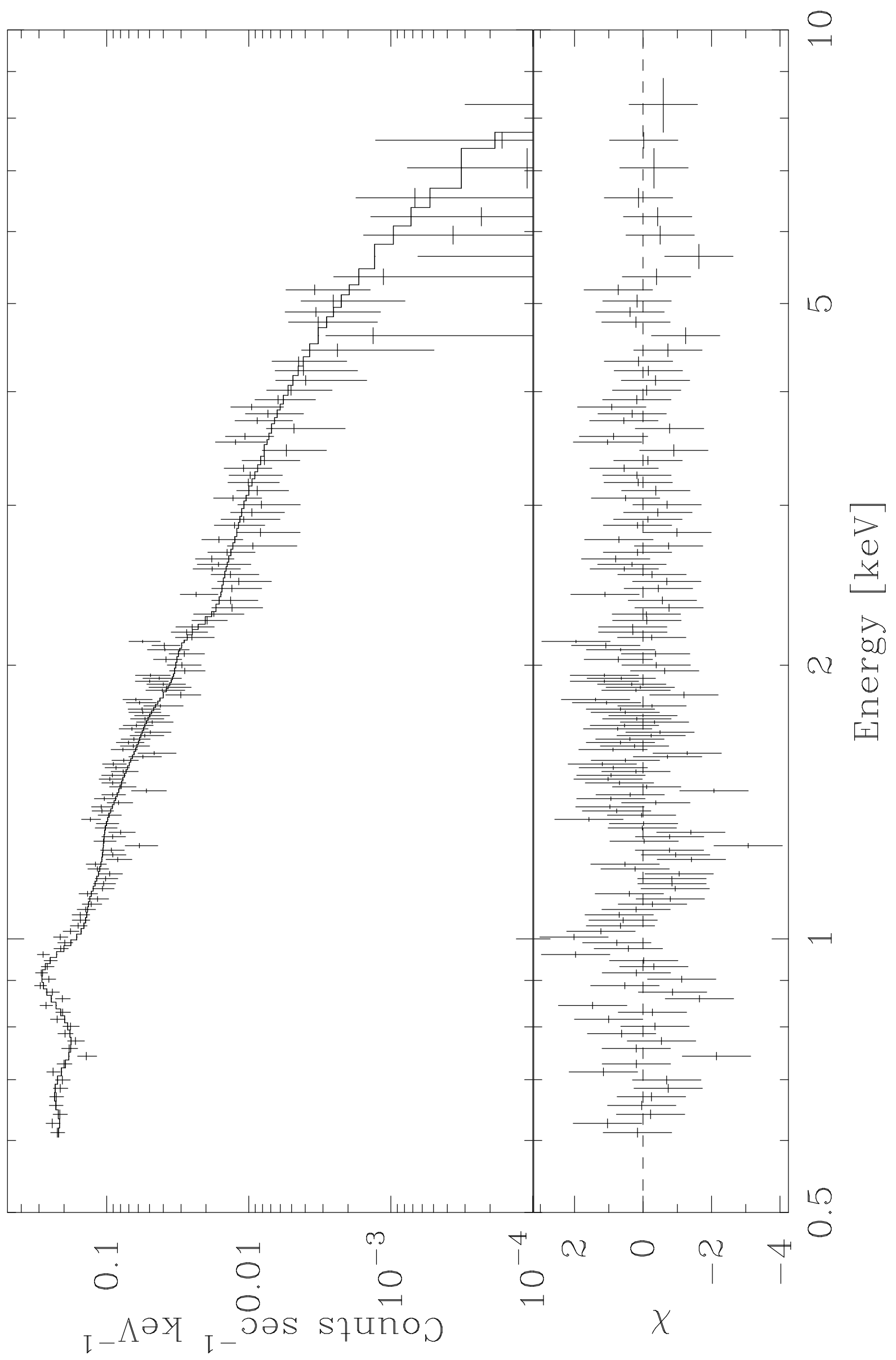

